\title{
Absorption Measurements in Microfluidic Devices using Ring-down Spectroscopy
}

\author{
Nicholas R. Trefiak, Jack Barnes, Fiona Rask, Daniel G. Courtney, \\ Richard Walford, Runkai Li, Richard D. Oleschuk, and \\ Hans-Peter Loock* \\ Department of Chemistry, Queen's University, Canada K7L $4 M 8$
}

\begin{abstract}
When monitoring separation events in microfluidic devices, one frequently needs to detect small amounts of analyte in picolitre sized volumes with a time response of milliseconds. Fluorescence detection is typically the method of choice due to its very high sensitivity and fast response. However, since many analytes are not naturally fluorescent, labelling protocols may have to be introduced and thereby increase the complexity of the analysis.

Here, we present an alternative method that is based on optical absorption, or more specifically on the ring-down time of an optical signal in a cavity or loop made of waveguide material. This optical decay constant changes as small liquid samples containing absorbing species are introduced into a fiber-optic loop. It is demonstrated that one can obtain the optical decay constant using a continuous wave laser beam that is intensity modulated and then coupled into an optical fiber loop. The inherent exponential decay in the fiber loop introduces a phase shift of the light emitted from the loop with respect to the pumping beam. By measuring this phase shift, one can readily determine the concentration of the analyte introduced between the two fiber ends and a model is established to describe the relationship. It is demonstrated that this technique, dubbed "phase-shift fiber-loop ring-down spectroscopy" (PS-FLRDS), is well suited as an absorption detector for any flow system in which the optical absorption path is limited by the instrument architecture.
\end{abstract}

Keywords: Fiber-loop ring-down spectroscopy (FLRDS), phase-shift, cavity ring-down (CRD), capillary electrophoresis (CE), microfluidics, absorbance detection.

\footnotetext{
* electronic mail: hploock@chem.queensu.ca

Facsimile: (613) 5336669 Photonic Applications in Biosensing and Imaging, edited by Warren C. W. Chan, Kui Yu, Ulrich J. Krull, Richard I. Hornsey,
Brian C. Wilson, Robert A. Weersink, Proc. of SPIE Vol. 5969, 59690O, (2005) $\cdot 0277-786$ X/05/\$15 doi: 


\section{INTRODUCTION}

In recent years microfluidic separation techniques have changed the face of analytical chemistry. For example capillary electrophoresis (CE) has greatly enhanced the sequencing of the human genome ${ }^{1}$ and microfluidic chips have permitted not only the chemical separation of nanoliter sized complex mixtures ${ }^{2}$ but also have been used in highthroughput chemical reactions. ${ }^{3}$ The main advantage of microfluidic devices, the small sample volume and high separation speed, poses a considerable challenge for the detection systems. Currently, fluorescence detection and UVvis absorption are the most frequently used methods used for capillary electrophoresis, with mass spectrometry forming an expensive but valuable alternative for extremely complex mixtures. ${ }^{4}$

For label-free detection, UV-vis absorption has been the most common as a result of its universality and sensitivity $\left(10^{-5}\right.$ to $\left.10^{-6} \mathrm{M}\right){ }^{5}$ Capillaries used for CE typically have an inner diameter of about $100 \mu \mathrm{m}$, and microfluidic channels have typically dimensions of $30 \mu \mathrm{m} \times 50 \mu \mathrm{m}$. This results in poor sensitivity for analytes with low molar extinction coefficients due to the short absorption path lengths. ${ }^{6 ; 7}$ A conceptually simple solution is the introduction of multipass absorption cells. However, this poses a formidable engineering challenge, since ' $\mathrm{Z}$ ' ${ }^{8 ; 9}$ or bubble ${ }^{10 ; 11}$ cells - while simple to interface - result in loss of temporal resolution and therefore loss in separating power. Conversely, multireflection nanoliter-scale cells require exact and robust alignment of a highly collimated light source. Furthermore, all traditional absorption techniques suffer from problems associated with measuring small intensity changes in the presence of a relatively large intensity background. This problem is compounded by the fact that lasers as intense light sources may exhibit intensity fluctuations with time and laser wavelength. Not surprisingly, the lack of sensitivity and robustness is still considered the fundamental limitation of absorption detection techniques in microfluidic applications.

A similar problem in gas-phase absorption spectroscopy has been elegantly addressed by cavity ring-down spectroscopy (CRDS), ${ }^{12-16}$ a multipass absorption technique which is largely insensitive to light intensity fluctuations, since the optical loss (absorption) is inferred from the rate of the intensity change. Typically, a CRDS system consists of a pulsed laser as a light source, a cavity composed of two highly reflective mirrors and a sensitive light detector (e.g. a photo multiplier tube). After the laser pulse has been introduced into the cavity the rate of intensity loss inside the cavity is dependent on the quality of the mirrors and the presence of optical absorbers in the cavity. From an exponential fit to the envelope of the intensity of the light emitted from the cavity one can then determine the exponential decay time (ring-down time: RDT). This RDT depends only on the optical loss processes inside the cavity, and ideally not on the intensity of the incoming laser light pulse. Most importantly in a cavity filled with an absorbing medium the sensitivity and detection limit of the absorption measurement is enhanced by the increased effective absorption path length, where the sensitivity of the RDT measurement is largest when the optical absorption approaches zero. ${ }^{17}$ For very high finesse cavities effective absorption paths of several tens of kilometers have been achieved with benchtop instruments. ${ }^{14}$

More recently, the potential of CRDS in the detection and spectroscopy of analytes in condensed samples has been realized. Recent developments include deposition of a thin film onto the mirror surfaces ${ }^{18}$, situating a thin film in the cavity perpendicular to the cavity axis, ${ }^{19,20}$ at Brewster's angle, ${ }^{21}$ or using a monolithic crystal ${ }^{22-25}$ or a long optical waveguide loop ${ }^{26-29}$ as a cavity to probe the absorption by the evanescent wave. Many of these techniques have been the subject of a recent review. ${ }^{30}$

A technique similar to CRDS is fiber-loop ring-down spectroscopy (FLRDS), ${ }^{28 ; 29}$ in which a fiber-loop is used instead of the mirrors to construct a ring-down 'cavity'. Nanosecond laser pulses are injected into the fiber, and can be observed for typically 100-200 round trips. With each roundtrip the laser pulse passes through the sample, which is situated between the ends of the optical fiber waveguide. The fractional loss in laser intensity is detected by fast photodetector. Recently, a refined technique, phase-shift FLRDS, was demonstrated to give a faster time response and lower detection limit in the detection of small amount liquid sample. ${ }^{31}$ Similar to the equivalent gas-phase technique, ${ }^{32}$ in PS-FLRDS an intensity-modulated continuous wave $(\mathrm{cw})$ laser with angular modulation frequency, $\Omega$, illuminates the fiber loop. The phase difference between the light entering the loop and exiting the loop is then related to the ringdown time $\tau$ by $\tan (\Delta \phi)=-\tau \Omega$.

Phase angle measurements have considerable advantages over pulsed RDT measurements: the method has a time resolution that can be a high as $1 \mathrm{kHz}$., the device can be made very compact, the data requires no fitting, and, since the duty cycle is close to $50 \%$, weak light sources $(P<10 \mathrm{~mW})$ are sufficient. 
On the other hand, it is not readily intuitive whether reliable phase shift measurements can be made when the optical decay is not a single exponential. In loops made of multimode fibers, multi-exponential decays are a concern, since comparable intensity is carried in the cladding and core of the fiber, and the intensity of the cladding and core modes decay at different rates. In this paper, we characterize multi-exponential decays in fiber cavities using phase angle measurements and illustrate how the individual decay constants may be obtained. For this purpose a linear stretch of single mode fiber was made into a cavity using fiber Bragg gratings (FBG's) ${ }^{33}$ and it is shown that its ring-down time can be inferred from phase shift measurements.

We also present a simple interface of the fiber loop to a capillary and characterize the detection sensitivity and detection speed. Finally, an improved coupling method of the fiber loop to the capillary is introduced by using a lensed fiber. This configuration is then compared to the previously presented straight-cut fiber ends.

\section{EXPERIMENTAL}

\subsection{Fiber cavity}

As shown schematically in Fig. 1 a fiber cavity is formed by two highly reflective fiber Bragg gratings (Avensys, Pt. Claire, QC) fabricated at the ends of a single $70 \mathrm{~m}$ piece of single-mode fiber (Corning, SMF-28). An intensitymodulated $c w$ laser (Ando AQ4320D) operated at around $1530 \mathrm{~nm}(4 \mathrm{~mW})$ was used as a laser source. The laser is modulated in a square wave using the internal chop between 30-300 kHz. Output from the reference chop waveform was used as the reference for the lock-in amplifier. A 99:1 (Lightel Technologies Inc.) 2/1 directional coupler was used as an optical isolator to the FBG cavity. The light emitted from the cavity is detected by an InGaAs detector (D400FC, ThorLabs Inc, Newton, NJ, USA). The signal is analyzed using either a lock-in amplifier (SRS $844,30 \mathrm{kHz}-200 \mathrm{Mhz}$, accuracy $\delta \phi=0.05$ $\mathrm{deg}$ ) or a homebuilt preamplifier/phase-comparator circuit (Analog Devices, $\delta \phi=0.1 \mathrm{deg}$ ). The offset phase shift changes slightly with the modulation frequency and we measure this dependence using a patchcord between the laser and the detector before subtracting it from the experimental data.

\subsection{Fiber loop}

An $80 \mathrm{~m}$ long multimode optical fiber (AS100/140 IRPI, Fibertech Optica Inc. GER.) was used to make a fiber loop (Fig. 1). The two fiber ends were aligned in a microcross

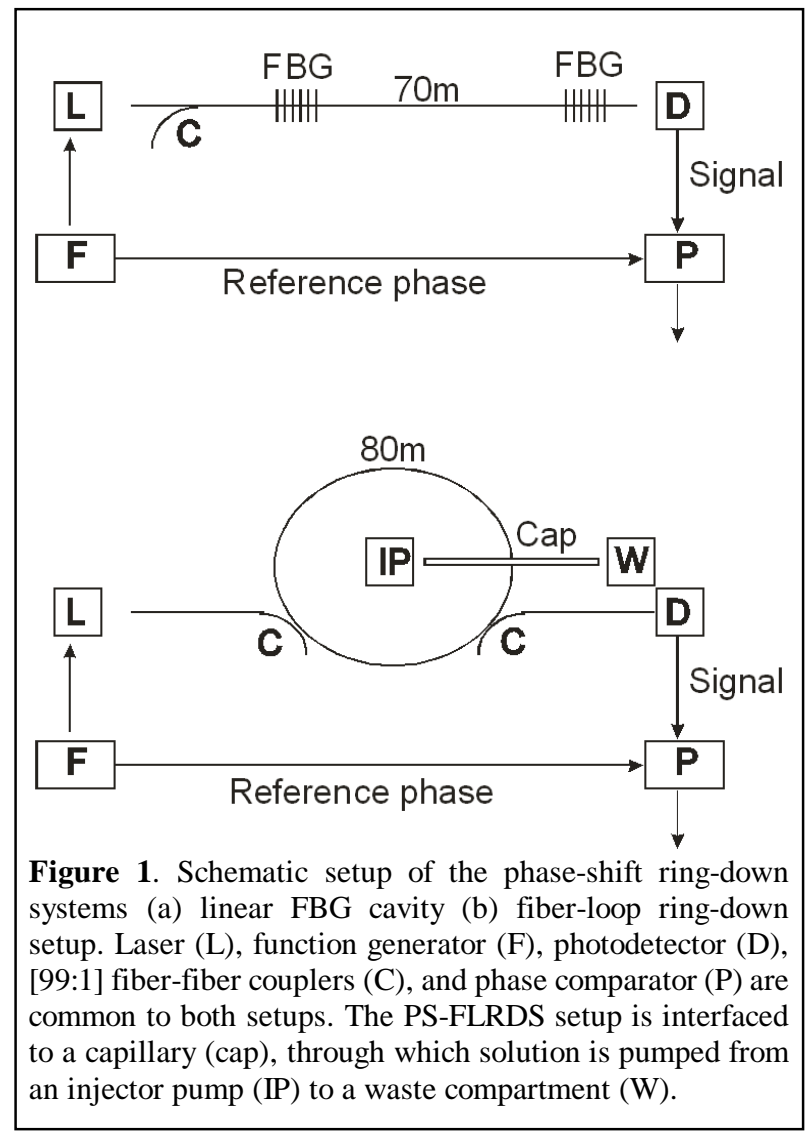
(P.777 MicroCross, Upchurch Scientific, USA) and the distance between them was adjusted to about $150 \mu \mathrm{m}$. Two pieces of fused silica capillary (I.D.100 $\mu \mathrm{m} /$ O.D.360 $\mu \mathrm{m}$, Polymicro Technologies, Phoenix, AZ., USA) were introduced and fixed in the other microcross channel. A 6-port injector with a $2-\mu \mathrm{L}$ inner sample loop and a syringe pump (Harvard Apparatus, Holliston, MA. USA) were used to inject sample solution and drive the flow (10 $\mu \mathrm{L} / \mathrm{min})$, respectively.

The laser diode (JDS Uniphase SDL-2372-P1, $810 \mathrm{~nm} \pm 3 \mathrm{~nm}$, max. $2 \mathrm{~W}$ ) is current-modulated at $100 \mathrm{kHz}$. To couple the laser light into the optical fiber loop, the laser output is focused onto the end of a $1 \mathrm{~m}$ optical high power delivery fiber (AS400/440 IRPI, Fibertech Optica Inc. GER.) This fiber was used to introduce the light into the loop by illuminating a small section of the fiber loop. Index matching gel (ThorLabs Inc. Newton, NJ, USA) was used to couple the laser light into the fiber loop. A photomultiplier tube (Hamamatsu 950) is placed at a different location in the loop and monitors the emission from the loop by detection of photons scattered from the fiber. The PMT signal is 
then fed into a fast lock-in amplifier (Stanford Research Systems, SR 844) and referenced to the driving current of the laser diode. To reduce radio frequency interference, all the cables are shielded. The laser dye ADS805(American Dye Source) was used without further purification and had an absorption coefficient of $\varepsilon=\ln (10) 1.6 \times 10^{5} \mathrm{M}^{-1} \mathrm{~cm}^{-1}$.

The data acquisition rate was set to $3.33 \mathrm{~Hz}$, which provided a sufficient sampling frequency for chromatographic measurements and permitted time integration over about 60,000 cycles. As shown below, $-1 / \tan \left(\phi-\phi_{0}\right)$ is linearly proportional to the concentration and therefore can be used directly to obtain an electropherogram.

\subsection{Fabrication of fiber lenses}

A fusion splicer (Fitel S182) was used to create lensed surfaces on the cleaved ends of multimode fiber (AS100/140 IRPI, Fibertech Optica Inc. GER.). The fiber was aligned perpendicular to the fusion splicer electrodes while the distance from the electrodes was varied. Using the default arc settings (used for splicing multimode fiber), a bulging curved surface was created. In order to create curved surfaces without bulging, various combinations of arc time, power and perpendicular distance from electrodes were attempted. It became clear that two methods could be use to create near hemispherical surfaces to channel and focus emitted light.

Using $88 \%$ of the maximum power several arcs (3-12) lasting about $100 \mu$ s produced optimal results with the fiber end positioned approximately $180 \mu \mathrm{m}$ from the electrodes. Longer arc times combined with low arc power settings also proved effective. An arc lasting $10 \mathrm{~ms}$ set to $12 \%$ of maximum power created similar results to the above method when the fiber end was positioned directly between the electrodes. However, the timing/power variations of the fusion splicer performance made the lenses produced by this method less predictable compared to the "multiple arc" method.

With these settings five near hemispherical fiber ends, with a radius of $76 \pm 4 \mu \mathrm{m}$ were made reproducibly. The average widening (bulge) of the fiber ends was $109 \%$ of the waveguide diameter $(140 \mu \mathrm{m})$. The focussing of the guided core modes was observed using a $632 \mathrm{~nm}$ HeNe laser (Melles Griot 05-LLR811) free space coupled into lensterminated and straight-cut lengths of fiber. The exiting beam was immersed in a dilute milk solution and imaged using a microscope (Nikon Eclipse ME600).

\section{RESULTS AND DISCUSSION}

\subsection{Phase-shift ring-down spectroscopy in a FBG-cavity}

Two devices were used in the present work. The fiber cavity formed by FBGs was made of single mode fiber into which 1.53 micron light was coupled, whereas the fiber loop was made of multimode fiber into which $810 \mathrm{~nm}$ light was coupled. Both "cavities" had a quasi continuous mode structure, due to the very large number of axial modes supported by the fiber loop, and the length and comparably low finesse of the linear cavity, respectively. In both cases the ring-down time is described by ${ }^{29}$

$$
\tau=\frac{L n}{c_{0}\left(\sum_{i} \varepsilon_{i} C_{i} d-\ln T_{\text {splice }}+\varepsilon_{\text {fiber }} L\right)}
$$

where $L$ is the length of the cavity/loop, $n$ is the refractive index of the waveguide material, $c_{0}$ is the vacuum speed of light, $T$ is the transmission of the fiber-fiber coupling of the loop, and $\varepsilon_{\text {fiber }}$ is the absorption coefficient of the fiber material given with respect to base e. Should the cavity include a section into which analyte is introduced, additional terms are introduced for the absorption coefficient, $\varepsilon_{i}$ and concentration, $C_{i}$ of each of the compounds and the width of the gap, $d$.

The optical decay or build-up constant of such a cavity, $\tau$, is typically measured in the time domain. Alternatively the ring-down time can be inferred from measurements of the phase shift between intensity-modulated light entering and exiting the cavity. ${ }^{31}$ The phase-shift, $\phi$ and ring-down time, $\tau$, are related simply by ${ }^{34}$

$$
\phi-\phi_{0}=-\arctan (\Omega \tau)
$$

where $\phi_{0}$ is the phase angle in the absence of analyte and $\Omega$ is the angular modulation frequency of the $c w$ laser. The offset angle $\phi_{0}$ contains all contributions due to the inevitable delays in the signal transmission and processing. 


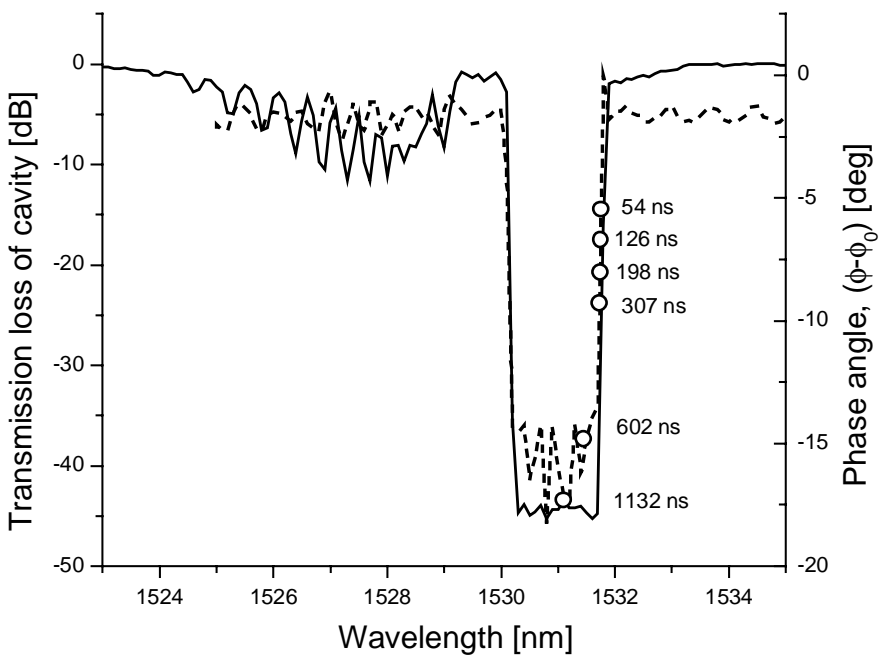

Figure 2: Transmission spectrum of the FBG cavity recorded with an optical spectrum analyser, (solid curve) and using phase angle measurements (dashed curve). Ring down times were derived at six wavelengths using linear fits to the data in Figure 3 and are included as circles. For a single exponential decay $\tau=1.2 \mu$ s corresponds to a reflection of $8 \mathrm{~dB}$ for each FBG, i.e. a finesse of about 15 .
Equation (2) illustrates that the ring-down time is proportional to $\tan \left(\phi_{0}-\phi\right)$. Considering that $1 / \tau$ is linearly dependent to the concentration of analyte between the two fiber ends of the fiber loop, a linear relationship also exists between $1 / \tan \left(\phi_{0}-\phi\right)$ and concentration. An electropherogram / chromatogram can then be constructed simply by recording $1 / \tan \left(\phi_{0}-\phi\right)$ as a function of time. As shown in previous work the time response of phase shift FLRDS is greatly improved compared to pulsed FLRDS - typically from tens of seconds to milliseconds. ${ }^{31}$ In the present study, the data-acquisition time was set to $300 \mathrm{~ms}$, as a compromise between high number of averaged cycles (high $\mathrm{S} / \mathrm{N}$ ratio) and a fast acquisition rate, which is comparable to the response of a conventional UV-vis detector.

While it is convenient to obtain a measure for the optical loss in the cavity/loop simply by phase comparison, there may be a complication, if the cavity decay is not a single exponential function. Previously it was shown that multimode fiber-loops show

two distinct decay rates - a slow decay associated with the core modes and a fast decay associated with the cladding modes. ${ }^{29}$ The time response of such a loop is readily fit by a bi-exponential function, thus giving two ring-down times. The same loop interrogated by phase angle measurements will on the other hand produce only a single phase shift value which depends on both decay rates.

One can nevertheless infer both decay constants by analyzing the dependence of the phase shift on the modulation frequency. Here, we make use of the fact that at high modulation frequency the modulation depth associated with the slow decay approaches zero, and that a plot of $\tan (\phi)$ versus $\Omega$ will therefore have an asymptotic slope of $-\tau_{\text {fast }}$. As illustrated before ${ }^{34}$ the intensity emitted from a cavity/loop is given by

$$
I(t)=I_{0}\left(1+\frac{\alpha}{\sqrt{1+\Omega^{2} \tau^{2}}}[\sin \Omega t-\arctan (\Omega \tau)]\right)
$$

where $\alpha$ is the modulation depth of the incoming light. We assume that for two independent decay processes the emitted light can be described by the sum

$$
I(t)=I_{0}\left(1+\frac{\gamma \alpha_{1}}{\sqrt{1+\Omega^{2} \tau_{1}^{2}}}\left[\sin \Omega t-\arctan \left(\Omega \tau_{1}\right)\right]+\frac{(1-\gamma) \alpha_{2}}{\sqrt{1+\Omega^{2} \tau_{2}^{2}}}\left[\sin \Omega t-\arctan \left(\Omega \tau_{2}\right)\right]\right)
$$

where $0<\gamma<1$ describes the relative intensity associated with these two processes. While the phase shift of each component with respect to the input oscillation is given by eq. (2), the phase shift of the composite waveform in eq. (4) is not trivially derived. Figure 4 displays the dependence of the numerically determined phase angle as a function of the modulation frequency. We note that at high $\Omega$ the limiting slope is simply given by eq.(2) (where $\tau=\tau_{\text {fast }}$ ), while at slow modulation and assuming that $\alpha_{1}=\alpha_{2}$, both components contribute to the slope according to the fractional power carried in each, i.e.

$$
\tan \phi=-\Omega\left((1-\gamma) \tau_{2}+\gamma \tau_{1}\right)
$$

The graph may exhibit a minimum when the decay rates are considerably different from each other. 


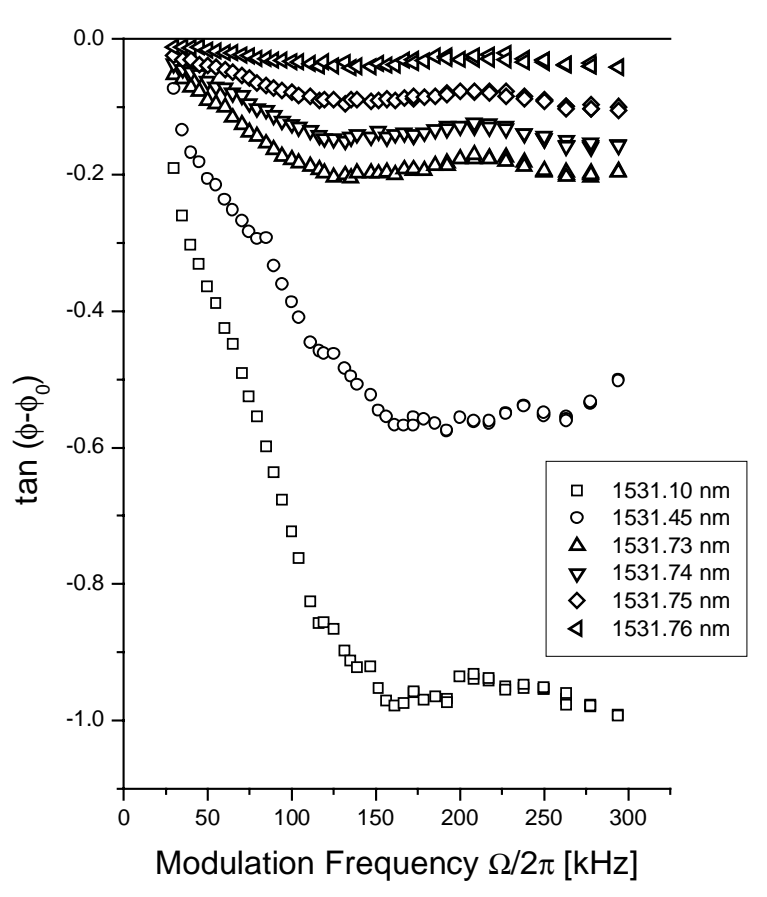

Figure 3 The phase shift of the intensity modulated light emitted by a FBG cavity recorded at six selected wavelengths. As expected from eq. 2 the curves are linear for low angular modulation frequencies.

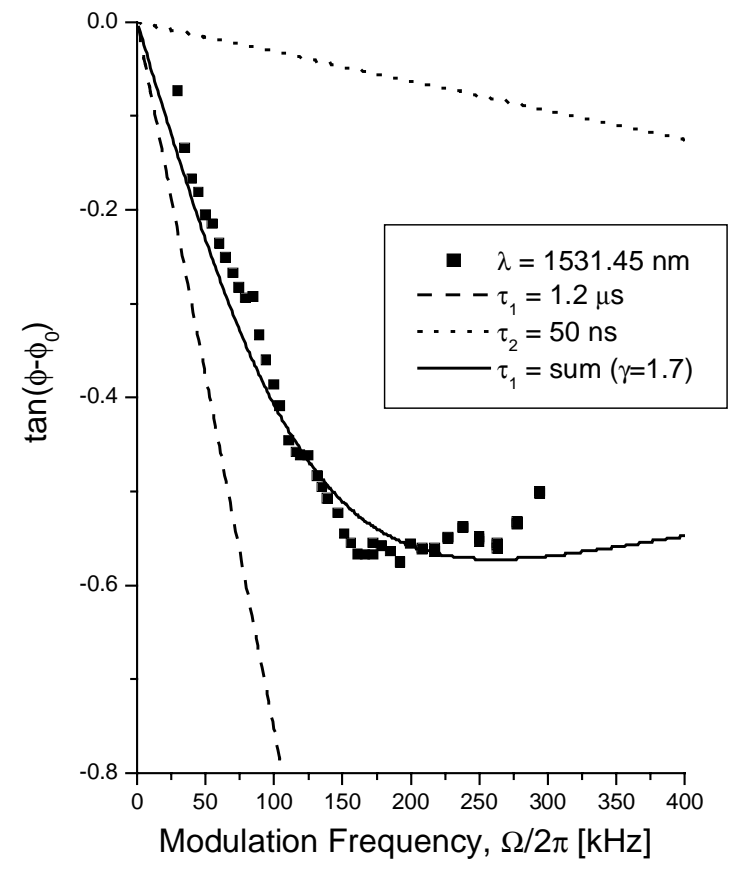

Figure 4: Cavity response curves at $1531.45 \mathrm{~nm}$. The slope of the linear portion at low angular modulation frequency $\Omega$ is given by $(\gamma-1) \tau_{2}-\gamma \tau_{1}$ which in this case is close to the negative ring-down time of the core modes, $-\tau_{1}$. The limiting slope for high $\Omega$ is given by $-\tau_{2}$.

Fig 2 shows the transmission spectrum of the FBG fiber cavity recorded with an optical spectrum analyzer (Agilent, 86142B). The spectrum can be reproduced by monitoring the phase angle difference between an intensity-modulated laser illuminating the cavity and the modulated light exiting the loop. Due to the length of the cavity $(L=70 \mathrm{~m}$, free spectral range $1.5 \mathrm{MHz})$ and the linewidth of the laser $(\sim 1 \mathrm{MHz})$ numerous cavity modes were excited. At several selected wavelengths near the transmission edge of the FBGs the modulation frequency was ramped between $30 \mathrm{kHz}$ and $300 \mathrm{kHz}$ and the phase angle response is displayed in Fig 3. It is apparent that for the lowest modulation frequencies the curves are linear with slopes that yield the composite ring-down time of two or more decays. Fig 4 shows exemplarily that these decays may be approximated by two optical loss processes with decay rates of $\tau_{1}=1.2 \mu \mathrm{s}$ and $\tau_{2}=50 \mathrm{~ns}$ and $\gamma=1.7$. All other curves could be approximated with the same RDT's and values for $\gamma$ between $\gamma=$ 5 (at $1531.10 \mathrm{~nm}$ ) and $\gamma=0.03$ (at $1531.76 \mathrm{~nm}$ ).

The time response of such a system is ultimately limited by the inverse of the modulation frequency but is for practical purposes much slower. It was found that good $\mathrm{S} / \mathrm{N}$ may be obtained by setting the acquisition rate to $10 \mathrm{~Hz}$, which is more than sufficient for most analytical applications.

\subsection{Phase-shift ring-down spectroscopy in a fiber loop}

Using the definition of the ring-down time Eq. (2) can be rewritten ${ }^{31}$

$$
\phi=\phi_{0}-\arctan \frac{n \Omega L}{c_{0}(A+\varepsilon d C)}
$$

Values for $d, A$ and $\phi_{0}$ were determined by fitting of the concentration dependence of the phase angle, which was obtained by suspending a drop of dye solution between two fiber ends held in a translation stage (see Figure 5). The 


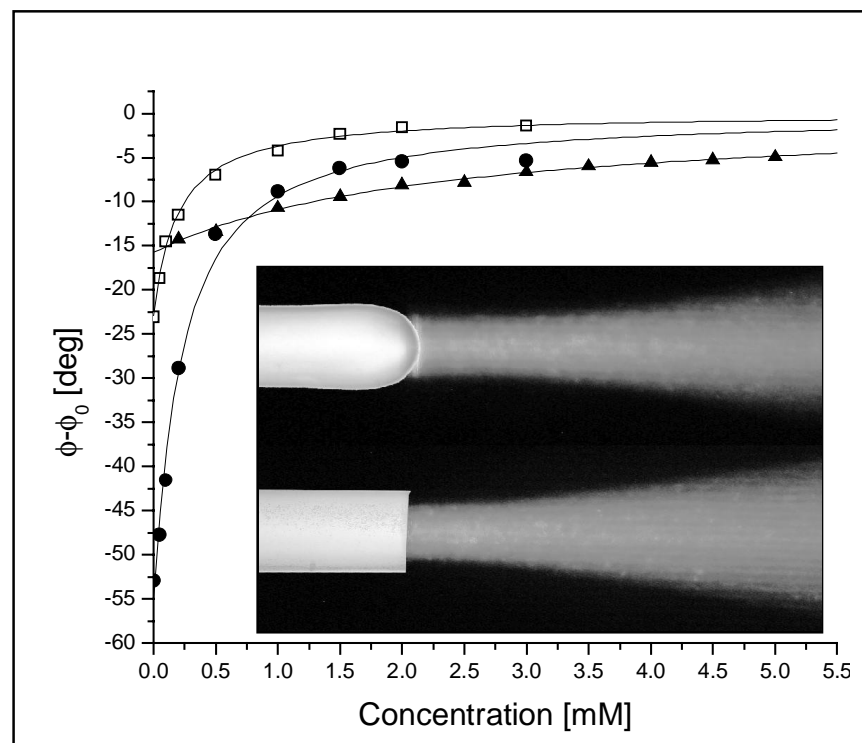

Figure 5. Phase-angle measurement as a function of concentrations. Shown are phase shifts for the lensed fiber ends in a translation stage (solid circles), straight cut fiber ends in a translation stage (open squares), and straight-cut fiber ends in a microcross (solid triangles). The curves are fits using equation (6) and fitting parameters $A, C, \phi_{0}$ and $d$, are given in the text. The insert shows a composite photograph of the two types of fiber ends illuminating a diluted milk emulsion.

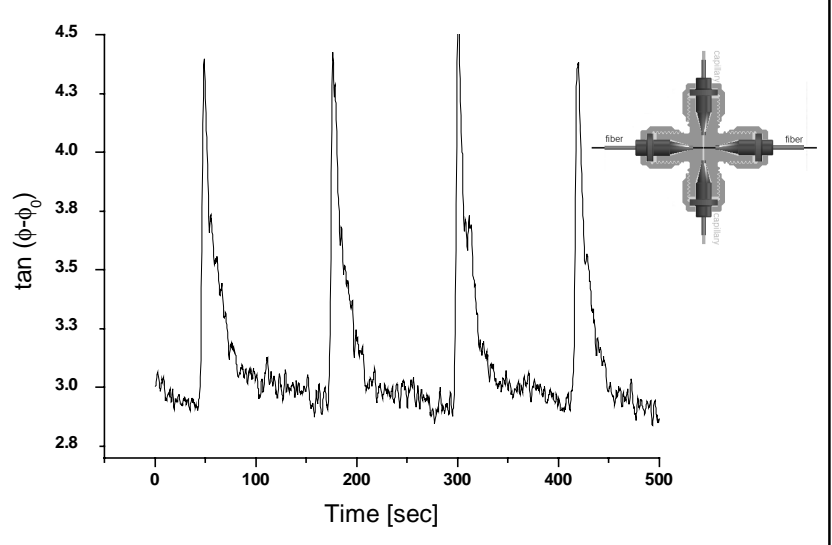

Figure 6. Flow trace for $2 \mathrm{mM}$ ADS805 dye in water probed by PS-FLRDS at $810 \mathrm{~nm}$ interfaced using the micro-cross shown in the insert. The injection volume was $2 \mu \mathrm{l}$, the flow rate was $10 \mu \mathrm{l} / \mathrm{min}$, and the acquisition rate was $3.33 \mathrm{~Hz}$. The relative standard deviation of peak areas is $6.07 \%$

straight-cut fiber ends. offset angle reflects the time delays in signal acquisition and processing and while the value is constant for each configuration it is difficult to predict. Two different loops were used: for one interface the fiber ends were cleaved and inspected for smoothness and planarity, whereas for the other interface the emitting fiber was curved into a lens to permit larger transmission into the receiving fiber. The cyanine dye ADS805WS was selected as an analyte to test the performance factors of the PSFLRDS system because the maximum absorption wavelength of the dye $(807 \mathrm{~nm})$ is close to the laser wavelength $(810 \mathrm{~nm})$.

For the two interfaces the fit gave similar values for $\phi_{0}$ $\approx 25 \mathrm{deg}$ and values for $d$ (straight $)=86 \mu \mathrm{m}$ and $d$ (lens) $=35 \mu \mathrm{m}$, that correspond to interaction volumes of 675 and 275 femtoliters, respectively. On the other hand, the values for $A=0.58$ and 0.17 , for the straight-cut and lensed fibers, respectively, are dramatically different from each other indicating that the lensed fiber interface has a much improved coupling efficiency. Assuming that the value for the fiber attenuation, $\varepsilon_{\text {fiber }}=0.0078 \mathrm{~m}^{-1}(3.4 \mathrm{~dB} / \mathrm{km}$ given by the manufacturer's specifications) is the same for both loops, one can from $A=-\ln T+\varepsilon_{\text {fiber }} L$ readily determine the coupling efficiency of $T_{\text {(straight) }}=0.60$ and $T_{\text {(lens) }}=0.90$. The ring-down times associated with these background losses, i.e. at $C=0$, are $\tau=670 \mathrm{~ns}$ and $2.3 \mu \mathrm{s}$, respectively. One can show that the larger transmission for the lensed fiber end is largely due to the focusing effect, and not due to the difference in the size of the gap.

From the fitting parameters we further estimate the detection limit and dynamic range of the concentration measurements. With a measurement accuracy of $\delta \phi=$ $1 \mathrm{deg}$ the detection limit for the lensed setup is $5 \mu \mathrm{M}$ for ADS805 dye, i.e. $\varepsilon C_{\min }=0.8 \mathrm{~cm}^{-1}$. At the instrumental limit of $\delta \phi=0.05 \mathrm{deg}$ the detection limit corresponds to $240 \mathrm{nM}\left(\varepsilon C_{\min }=0.04 \mathrm{~cm}^{-1}\right)$. This is a considerable improvement over the straight cut fiber $10 \mu \mathrm{M}\left(\varepsilon C_{\min }=1.6 \mathrm{~cm}^{-1}\right)$ at $\delta \phi=1 \mathrm{deg}$ and $450 \mathrm{nM}$ $\left(\varepsilon C_{\min }=0.08 \mathrm{~cm}^{-1}\right)$ at $\delta \phi=0.05 \mathrm{deg}$. The dynamic range can be calculated using the same measurement accuracy of $\delta \phi=1 \mathrm{deg}$ and is $10 \mathrm{mM} / 5 \mu \mathrm{M}=2000$ for the lensed fiber and $4 \mathrm{mM} / 10 \mu \mathrm{M}=500$ for the

\subsection{Microcross interface}

While mechanical splices and translation stages are suitable for proof-of-concept measurements, a rugged and inexpensive fiber / capillary interface has to be used in any routine analytical microfluidic application. Figure 6 shows a commercial micro-cross that accepts four fibers or capillary ends with an outer diameter of about 120-150 $\mu \mathrm{m}$. A 
simple interface is made by inserting the fiber ends to within $5-50 \mu \mathrm{m}$ of each other and then inserting the capillary ends. The dead volume of such a device is $38 \mathrm{~nL}$, but acceptable for many applications.

A series of $2 \mu \mathrm{L}$ samples of with different concentrations of ADS805WS dye $(0.2-5.0 \mathrm{mM})$ was injected into the microcross detection gap $(d=7.8 \mu \mathrm{m}$ obtained from the fit in Fig 5) and a typical flow trace is shown in the figure 6. Fair repeatability of peak areas was obtained with a relative standard deviation of $6.07 \%$. Using three times the baseline noise, the limit of detection (LOD) of the micro-cross PS-FLRDS system can be calculated as $200 \mu \mathrm{M}$. While this is worse compared to the detection limits reached with the translation stages, the higher detection limit is likely due to a misalignment of the fiber ends in this preliminary experiment. The fiber is held only by four screw fittings and the microcross consequently does not permit accurate alignment of the fiber ends. Finally, the dead volume of the microcross is $38 \mathrm{~nL}$, which is comparably large and will affect the separation efficiency and hence indirectly the sensitivity of the concentration measurement. Future work will therefore focus on the development of a different fiber/capillary interface, which allows for easier and more reproducible alignment of the fiber ends. Also we will incorporate the lensed fiber ends into the loop and thus hope to enhance the detection efficiency even further.

\section{CONCLUSION}

Phase-shift FLRDS is demonstrated to be a viable and simple alternative to time resolved ring-down spectroscopy in optical waveguides. Here the fiber cavity or fiber loop is driven by a periodically oscillating laser field and the phase shift of the light emitted from the loop with respect to the incoming light, is used to infer the optical loss in the fiber. Complications may arise if there exists more than one loss process. While these decay processes can be readily separated in the time domain using a fit to a multi-exponential decay, phase-shift characterization of competing loss processes is less obvious. Nevertheless, we demonstrate that a biexponential decay leads to a characteristic response of the phase angle, $\phi$, (or rather $\tan \left(\phi-\phi_{0}\right)$ ) to the modulation frequency. This curve can be used to determine the two ringdown times from the limiting behavior at high and low angular modulation frequency. As demonstrated such a response is obtained when interrogating a fiber cavity in which the light is confined by two fiber Bragg gratings.

Fiber-loops made of multimode fiber form a special "cavity", which has no mode structure, and lends itself to absorption measurements of sub-picoliter liquid volumes. Again, phase-shift measurements provide the time response $(\sim 100 \mathrm{~ms})$ and detection limit needed for separation and analysis of microliter-sized samples in microfluidic channels. We illustrate that the coupling efficiency, detection limit and dynamic range can be improved by using lensed fibers. Preliminary experiments using a commercial micro-cross, to hold the fiber ends and the capillary, point towards an application of PS-FLRDS in capillary electrophoresis. An electropherogram was obtained from the temporal evolution of $1 / \tan (-\phi)$. The reproducibility of the measurement and its response time make this interface amenable to chromatographic and electrophoretic systems where limited path length is a concern. On the other hand, it is apparent that the micro-cross has a detection limit which is presently too high, indicating that precision devices may be needed to ensure good alignment of the fiber ends.

We note that the cyanine dyes used in this work are common noncovalent labels for the analysis of proteins and DNA. The PS-FLRDS technique presented here could hence be a potential sensitive detection method for analyzing proteins and DNA.

\section{ACKNOWLEDGMENTS}

The authors thank Dr. Zhaoguo Tong for many useful discussions and technical assistance and Dr. John Cartledge for generously providing the fusion splicer. We also acknowledge the Natural Sciences and Engineering Research Council of Canada, the Canadian Foundation for Innovation, the Ontario Innovation Trust, Premier's Research Excellence Award, Photonic Research Ontario and Queen's University for financial support. 


\section{REFERENCE LIST}

(1) Dovichi, N.; Zhang, J. Angew. Chemie Int.Ed. 2000, 39, 4463.

(2) Polson, N. A.; Hayes, M. A. Anal.Chem. 2001, 73, 312.

(3) Nagaki, A.; Togai, M.; Suga, S.; Aoki, N.; Mae, K.; Yoshida, J. J. Am. Chem. Soc. 2005, $127,1166$.

(4) Oleschuk, R. D.; Harrison, D. J. TRAC-Trends in Analytical Chemistry 2000, 19, 379-388.

(5) Landers, J.P. (ed.) Handbook of Capillary Electrophoresis; CRC Press: NY, 1997.

(6) Poppe, H. Anal. Chim. Acta 1980, 114, 59-70.

(7) Taylor, J. A.; Yeung, E. S. J. Chrom.atogr. 1991, 550, 831-837.

(8) Chervet, J. P.; Vansoest, R. E. J.; Ursem, M. J. Chromatogr. 1991, 543, 439-449.

(9) Wang, T. S.; Aiken, J. H.; Huie, C. W.; Hartwick, R. A. Anal. Chem. 1991, 63, 1372-1376.

(10) Lin, Y. W.; Huang, C. C.; Chang, H. T. Anal. Bioanal. Chem. 2003, 376, 379-383.

(11) Tseng, W. L.; Lin, Y. W.; Chen, K. C.; Chang, H. T. Electrophoresis 2002, 23, 2477-2484.

(12) O'Keefe, A.; Deacon, D. Rev. Sci. Instr. 1988, 59, 2544.

(13) Scherer, J. J.; O'Keefe, J. B. P.; Saykally, R. J. Chem. Rev. 1997, 97, 25.

(14) Wheeler, M. D.; Newman, S. M.; OrrEwing, A. J.; Ashfold, M. N. R. J. Chem. Soc. Faraday Trans. 1998, 94 , 337-351.

(15) Brown, S. S. Chem. Rev. 2003, 103, 5219-5238.

(16) Berden, G.; Peeters, R.; Meijer, G. Int. Rev. Phys. Chem. 2000, 19, 565-607.

(17) Jakubinek, M.; Tong, Z.; Manzhos, S.; Loock, H.-P. Can.J. Chem. 2004, 82, 873.

(18) Kleine, D.; Lauterbach, J.; Kleinermanns, K.; Hering, P. Appl. Phys. B-Lasers and Optics 2001, 72, $249-252$.

(19) Engeln, R.; von Helden, G.; van Roij, A. J. A.; Meijer, G. J. Chem. Phys. 1999, 110, 2732-2733.

(20) Logunov, S. L. Appl. Optics 2001, 40, 1570-1573.

(21) Muir, R. N.; Alexander, A. J. Phys. Chem. Chem. Phys. 2003, 5, 1279-1283.

(22) Pipino, A. C. R.; Hudgens, J. W.; Huie, R. E. Rev. Sci. Instr. 1997, 68, 2978-2989.

(23) Pipino, A. C. R.; Hudgens, J. W.; Huie, R. E. Chem. Phys. Lett. 1997, 280, 104-112.

(24) Pipino, A. C. R. Phys. Rev. Lett. 1999, 83, 3093-3096.

(25) Pipino, A. C. R.; Hoefnagels, J. P. M.; Watanabe, N. J. Chem. Phys. 2004, 120, 2879-2888.

(26) Tarsa, P. B.; Rabinowitz, P.; Lehmann, K. K. Chem. Phys. Lett. 2004, 383, 297-303.

(27) Tarsa, P. B.; Wist, A. D.; Rabinowitz, P.; Lehmann, K. K. Appl. Phys. Lett. 2004, 85, 4523-4525.

(28) Tong, Z.; Jakubinek, M.; Wright, A.; Gillies, A.; Loock, H.-P. Rev. Sci. Instrum. 2003, 74, 4818.

(29) Brown, R.; Kozin, I.; Tong, Z.; Oleschuk, R.; Loock, H.-P. J. Chem. Phys. 2002, 119.

(30) Vallance, C. New J. Chem. 2005, 29, 867-874.

(31) Tong, Z.; Wright, A.; McCormick, T.; Loock, H.-P. Anal. Chem. 2004, 76, 6594.

(32) Engeln, R.; vonHelden, G.; Berden, G.; Meijer, G. Chem. Phys. Lett. 1996, 262, 105-109.

(33) Gupta, M.; Jiao, H.; O'Keefe, A. Opt. Lett. 2002, 27, 1878-1880.

(34) Engeln, R.; vonHelden, G.; Berden, G.; Meijer, G. Chem. Phys. Lett. 1996, 262, 105-109. 